\title{
A CONCEPT OF SYNCHRONICITY ASSOCIATED WITH CONVEX FUNCTIONS IN LINEAR SPACES AND APPLICATIONS
}

\author{
S. S. DRAGOMIR
}

(Received 1 February 2010)

\begin{abstract}
A concept of synchronicity associated with convex functions in linear spaces and a Chebyshev type inequality are given. Applications for norms, semi-inner products and convex functions of several real variables are also given.
\end{abstract}

2000 Mathematics subject classification: primary 26D15; secondary 26D20, 46B05.

Keywords and phrases: convex functions, Gâteaux derivatives, norms, semi-inner products, synchronous sequences, Chebyshev inequality.

\section{Introduction}

The Jensen inequality for convex functions plays a crucial role in the theory of inequalities due to the fact that other inequalities such as the arithmetic-geometric mean inequality, Hölder and Minkowski inequalities, and Ky Fan's inequality can be obtained as particular cases of it.

Let $C$ be a convex subset of the linear space $X$ and $f$ be a convex real-valued function on $C$. If $\mathbf{p}=\left(p_{1}, \ldots, p_{n}\right)$ is a probability sequence and $\mathbf{x}=\left(x_{1}, \ldots, x_{n}\right) \in$ $C^{n}$, then

$$
f\left(\sum_{i=1}^{n} p_{i} x_{i}\right) \leq \sum_{i=1}^{n} p_{i} f\left(x_{i}\right)
$$

is well known in the literature as Jensen's inequality.

For refinements of the Jensen inequality and applications related to Ky Fan's inequality, the arithmetic-geometric mean inequality, the generalized triangle inequality and the $f$-divergence measures, see [5-11, 13-16, 21].

Assume that $f: X \rightarrow \mathbb{R}$ is a convex function on the real linear space $X$. Since for any vectors $x, y \in X$ the function

$$
g_{x, y}: \mathbb{R} \rightarrow \mathbb{R}, \quad g_{x, y}(t):=f(x+t y)
$$

(C) 2010 Australian Mathematical Publishing Association Inc. 0004-9727/2010 \$16.00 
is convex, it follows that the limits

$$
\nabla_{+(-)} f(x)(y):=\lim _{t \rightarrow 0+(-)} \frac{f(x+t y)-f(x)}{t}
$$

exist and are called the right (left) Gâteaux derivatives of the function $f$ at the point $x$ in the direction $y$.

It is obvious that, for any $t>0>s$,

$$
\begin{aligned}
\frac{f(x+t y)-f(x)}{t} & \geq \nabla_{+} f(x)(y)=\inf _{t>0}\left[\frac{f(x+t y)-f(x)}{t}\right] \\
& \geq \sup _{s<0}\left[\frac{f(x+s y)-f(x)}{s}\right]=\nabla_{-} f(x)(y) \\
& \geq \frac{f(x+s y)-f(x)}{s}
\end{aligned}
$$

for any $x, y \in X$ and, in particular,

$$
\nabla_{-} f(u)(u-v) \geq f(u)-f(v) \geq \nabla_{+} f(v)(u-v)
$$

for any $u, v \in X$. We call this the gradient inequality for the convex function $f$. It will be used frequently in the following in order to obtain various results related to Jensen's inequality.

The following properties are also of importance:

$$
\nabla_{+} f(x)(-y)=-\nabla_{-} f(x)(y)
$$

and

$$
\nabla_{+(-)} f(x)(\alpha y)=\alpha \nabla_{+(-)} f(x)(y)
$$

for any $x, y \in X$ and $\alpha \geq 0$.

The right Gâteaux derivative is subadditive while the left one is superadditive, that is,

$$
\nabla_{+} f(x)(y+z) \leq \nabla_{+} f(x)(y)+\nabla_{+} f(x)(z)
$$

and

$$
\nabla_{-} f(x)(y+z) \geq \nabla_{-} f(x)(y)+\nabla_{-} f(x)(z)
$$

for any $x, y, z \in X$. Some natural examples can be provided by the use of normed spaces.

Assume that $(X,\|\cdot\|)$ is a real normed linear space. The function $f: X \rightarrow \mathbb{R}$, $f(x):=\frac{1}{2}\|x\|^{2}$ is a convex function which generates the superior and inferior semiinner products

$$
\langle y, x\rangle_{s(i)}:=\lim _{t \rightarrow 0+(-)} \frac{\|x+t y\|^{2}-\|x\|^{2}}{t} .
$$


For a comprehensive study of the properties of these mappings in the geometry of Banach spaces see the monograph [10].

For the convex function $f_{p}: X \rightarrow \mathbb{R}, f_{p}(x):=\|x\|^{p}$ with $p>1$,

$$
\nabla_{+(-)} f_{p}(x)(y)= \begin{cases}p\|x\|^{p-2}\langle y, x\rangle_{s(i)} & \text { if } x \neq 0 \\ 0 & \text { if } x=0\end{cases}
$$

for any $y \in X$. If $p=1$, then

$$
\nabla_{+(-)} f_{1}(x)(y)= \begin{cases}\|x\|^{-1}\langle y, x\rangle_{s(i)} & \text { if } x \neq 0 \\ +(-)\|y\| & \text { if } x=0\end{cases}
$$

for any $y \in X$. This class of functions will be used to illustrate the inequalities obtained in the general case of convex functions defined on an entire linear space.

In the recent paper [12] the following refinement and reverse of the Jensen inequality in terms of the gradient were obtained.

THEOREM 1.1. Let $f: X \rightarrow \mathbb{R}$ be a convex function defined on a linear space $X$. Then for any n-tuple of vectors $\mathbf{x}=\left(x_{1}, \ldots, x_{n}\right) \in X^{n}$ and any probability distribution $\mathbf{p}=\left(p_{1}, \ldots, p_{n}\right) \in \mathbb{P}^{n}$ we have the inequality

$$
\begin{aligned}
\sum_{k=1}^{n} p_{k} \nabla_{-} f\left(x_{k}\right)\left(x_{k}\right)-\sum_{k=1}^{n} p_{k} \nabla_{-} f\left(x_{k}\right)\left(\sum_{i=1}^{n} p_{i} x_{i}\right) \\
\geq \sum_{i=1}^{n} p_{i} f\left(x_{i}\right)-f\left(\sum_{i=1}^{n} p_{i} x_{i}\right) \\
\geq \sum_{k=1}^{n} p_{k} \nabla_{+} f\left(\sum_{i=1}^{n} p_{i} x_{i}\right)\left(x_{k}\right)-\nabla_{+} f\left(\sum_{i=1}^{n} p_{i} x_{i}\right)\left(\sum_{i=1}^{n} p_{i} x_{i}\right) \geq 0 .
\end{aligned}
$$

A particular case of interest is for $f(x)=\|x\|^{p}$ where $(X,\|\cdot\|)$ is a normed linear space. Then for any $p \geq 1$, for any $n$-tuple of vectors $\mathbf{x}=\left(x_{1}, \ldots, x_{n}\right) \in X^{n}$ and any probability distribution $\mathbf{p}=\left(p_{1}, \ldots, p_{n}\right) \in \mathbb{P}^{n}$ with $\sum_{i=1}^{n} p_{i} x_{i} \neq 0$ we have the inequality

$$
\begin{aligned}
& \sum_{i=1}^{n} p_{i}\left\|x_{i}\right\|^{p}-\left\|\sum_{i=1}^{n} p_{i} x_{i}\right\|^{p} \\
& \quad \geq p\left\|\sum_{i=1}^{n} p_{i} x_{i}\right\|^{p-2}\left[\sum_{k=1}^{n} p_{k}\left\langle x_{k}, \sum_{j=1}^{n} p_{j} x_{j}\right\rangle_{s}-\left\|\sum_{i=1}^{n} p_{i} x_{i}\right\|^{2}\right] \\
& \quad \geq 0 .
\end{aligned}
$$

If $p \geq 2$ the inequality holds for any $n$-tuple of vectors and probability distribution.

Also, for any $p \geq 1$, for any $n$-tuple of vectors

$$
\mathbf{x}=\left(x_{1}, \ldots, x_{n}\right) \in X^{n} \backslash\{(0, \ldots, 0)\}
$$


and any probability distribution $\mathbf{p}=\left(p_{1}, \ldots, p_{n}\right) \in \mathbb{P}^{n}$ we have the inequality

$$
\begin{gathered}
p\left[\sum_{k=1}^{n} p_{k}\left\|x_{k}\right\|^{p}-\sum_{k=1}^{n} p_{k}\left\|x_{k}\right\|^{p-2}\left\langle\sum_{j=1}^{n} p_{j} x_{j}, x_{k}\right\rangle_{i}\right] \\
\geq \sum_{i=1}^{n} p_{i}\left\|x_{i}\right\|^{p}-\left\|\sum_{i=1}^{n} p_{i} x_{i}\right\|^{p} .
\end{gathered}
$$

For related inequalities for norms and inner products, see [1-4, 20, 22].

Motivated by the above results we introduce in this paper a class of sequences associated with convex functions in linear spaces and establish a Chebyshev type inequality and some new inequalities for convex functions. Applications for norms, semi-inner products and convex functions of several real variables are also given.

\section{2. $\nabla f$-Synchronicity}

Consider $f: X \rightarrow \mathbb{R}$ a convex function on the linear space $X$. We also assume that $u=\left(u_{1}, \ldots, u_{n}\right)$ and $v=\left(v_{1}, \ldots, v_{n}\right)$ are two $n$-tuples of vectors with $u_{i}, v_{i} \in X$, $i \in\{1, \ldots, n\}$.

Definition 2.1. We say that $v$ is $\nabla f$-synchronous with $u$ if

$$
\nabla_{-} f\left(u_{k}\right)\left(v_{k}-v_{j}\right) \geq \nabla_{+} f\left(u_{j}\right)\left(v_{k}-v_{j}\right)
$$

for any $k, j \in\{1, \ldots, n\}$. If the inequality is reversed in (2.1) for each $k, j \in$ $\{1, \ldots, n\}$, then we say that $v$ is $\nabla f$-asynchronous with $u$.

We notice that in general, if $v$ is $\nabla f$-asynchronous with $u$, this does not imply that $u$ is $\nabla f$-synchronous with $v$.

As general examples of such convex functions we can consider $f(x)=\|x\|^{p}$, $p \geq 1$, where $(X,\|\cdot\|)$ is a normed linear space. Since (see introduction)

$$
\begin{aligned}
& \nabla_{-} f(x)(y)=p\|x\|^{p-2}\langle y, x\rangle_{i} \quad \text { for } x, y \in X \text { with } x \neq 0 \text {; } \\
& \nabla_{-} f(0)(y)=\left\{\begin{array}{ll}
0 & \text { if } p>1 \\
-\|y\| & \text { if } p=1,
\end{array} \quad \text { for } y \in X ;\right. \\
& \nabla_{+} f(x)(y)=p\|x\|^{p-2}\langle y, x\rangle_{s} \quad \text { for } x, y \in X \text { with } x \neq 0 \text {; } \\
& \nabla_{+} f(0)(y)=\left\{\begin{array}{ll}
0 & \text { if } p>1 \\
\|y\| & \text { if } p=1,
\end{array} \quad \text { for } y \in X,\right.
\end{aligned}
$$

where $\langle\cdot, \cdot\rangle_{s}$ is the superior semi-inner product and $\langle\cdot, \cdot\rangle_{i}$ is the inferior semi-inner product, we can define the following concepts of synchronicity for the two $n$-tuples of vectors $u=\left(u_{1}, \ldots, u_{n}\right)$ and $v=\left(v_{1}, \ldots, v_{n}\right)$.

Let $p \geq 1$ and $u, v \in X^{n}$ be as above. We say that $v$ is $p-\nabla$-synchronous with $u$ if

$$
\left\|u_{k}\right\|^{p-2}\left\langle v_{k}-v_{j}, u_{k}\right\rangle_{i} \geq\left\|u_{j}\right\|^{p-2}\left\langle v_{k}-v_{j}, u_{j}\right\rangle_{s}
$$

for any $k, j \in\{1, \ldots, n\}$. 
We observe that for $p \in[1,2)$ we should assume that $u_{k} \neq 0$ for $k \in\{1, \ldots, n\}$. For $p=2$, the relation $(2.2)$ reduces to

$$
\left\langle v_{k}-v_{j}, u_{k}\right\rangle_{i} \geq\left\langle v_{k}-v_{j}, u_{j}\right\rangle_{s} \quad \text { for any } k, j \in\{1, \ldots, n\} .
$$

If $(X,\|\cdot\|)$ is a smooth normed space, meaning that the norm is Gâteaux differentiable on any $x \in X, x \neq 0$, and if we denote by $[\cdot, \cdot]$ the semi-inner product generating the norm $\|\cdot\|$ (see [10, pp. 19-20]), then the fact that $v$ is $p-\nabla$ synchronous with $u$ means that

$$
\left\|u_{k}\right\|^{p-2}\left[v_{k}-v_{j}, u_{k}\right] \geq\left\|u_{j}\right\|^{p-2}\left[v_{k}-v_{j}, u_{j}\right]
$$

for any $k, j \in\{1, \ldots, n\}$. For $p=2$,

$$
\left[v_{k}-v_{j}, u_{k}\right] \geq\left[v_{k}-v_{j}, u_{j}\right] \text { for any } k, j \in\{1, \ldots, n\} .
$$

Moreover, if the norm $\|\cdot\|$ is generated by an inner product $\langle\cdot, \cdot\rangle$, then $v$ is $p-\nabla$ synchronous with $u$ means that

$$
\left\langle v_{k}-v_{j},\left\|u_{k}\right\|^{p-2} u_{k}-\left\|u_{j}\right\|^{p-2} u_{j}\right\rangle \geq 0 \text { for any } k, j \in\{1, \ldots, n\},
$$

while for $p=2$, it reduces to

$$
\left\langle v_{k}-v_{j}, u_{k}-u_{j}\right\rangle \geq 0 \text { for any } k, j \in\{1, \ldots, n\},
$$

which is the concept of synchronous sequences in inner product spaces that was introduced in [18]. For some inequalities for synchronous sequences in inner product spaces, see $[17,18]$.

As natural examples of synchronous sequences in inner product spaces, we can consider the sequences $\left\{x_{i}\right\}_{i \in \mathbb{N}}$ and $\left\{A x_{i}\right\}_{i \in \mathbb{N}}$ where $A: X \rightarrow X$ is a positive linear operator on $X$, that is, $\langle A x, x\rangle \geq 0$ for any $x \in X$.

For a convex function $f: X \rightarrow \mathbb{R}$ we define $\tilde{\nabla} f(\cdot)(\cdot)$ as

$$
\tilde{\nabla} f(x)(y):=\frac{1}{2}\left[\nabla_{-} f(x)(y)+\nabla_{+} f(x)(y)\right],
$$

where $x, y \in X$. We observe that for $f$ as above, we have the homogeneity property:

$$
\tilde{\nabla} f(x)(\alpha y)=\alpha \tilde{\nabla} f(x)(y) \text { for any } x, y \in X,
$$

and any $\alpha \in \mathbb{R}$.

The following inequality for $\nabla-f$-synchronous sequences holds.

THEOREM 2.2. Assume that $v$ is $\nabla-f$-synchronous with $u$ and $\mathbf{p}=\left(p_{1}, \ldots, p_{n}\right)$ is a probability distribution. Then

$$
\sum_{i=1}^{n} p_{i} \tilde{\nabla} f\left(u_{i}\right)\left(v_{i}\right) \geq \sum_{i, j=1}^{n} p_{i} p_{j} \tilde{\nabla} f\left(u_{i}\right)\left(v_{j}\right) .
$$

PROOF. Since $\nabla_{+}(\cdot)(\cdot)$ is subadditive in the second variable, then

$$
\nabla_{+} f\left(u_{j}\right)\left(v_{i}-v_{j}\right) \geq \nabla_{+} f\left(u_{j}\right)\left(v_{i}\right)-\nabla_{+} f\left(u_{j}\right)\left(v_{j}\right)
$$


for any $i, j \in\{1, \ldots, n\}$. Also, by the fact that $\nabla_{-}(\cdot)(\cdot)$ is superadditive in the second variable, we have that

$$
\nabla_{-} f\left(u_{i}\right)\left(v_{i}\right)-\nabla_{-} f\left(u_{i}\right)\left(v_{j}\right) \geq \nabla_{-} f\left(u_{i}\right)\left(v_{i}-v_{j}\right)
$$

for all $i, j \in\{1, \ldots, n\}$. Now, by (2.11), (2.12) and by the definition of $\nabla-f$ synchronicity, we deduce that

$$
\nabla_{-} f\left(u_{i}\right)\left(v_{i}\right)-\nabla_{-} f\left(u_{i}\right)\left(v_{j}\right) \geq \nabla_{+} f\left(u_{j}\right)\left(v_{i}\right)-\nabla_{+} f\left(u_{j}\right)\left(v_{j}\right),
$$

which is equivalent to

$$
\nabla_{-} f\left(u_{i}\right)\left(v_{i}\right)+\nabla_{+} f\left(u_{j}\right)\left(v_{j}\right) \geq \nabla_{+} f\left(u_{j}\right)\left(v_{i}\right)+\nabla_{-} f\left(u_{i}\right)\left(v_{j}\right)
$$

for all $i, j \in\{1, \ldots, n\}$.

Therefore, by multiplying (2.13) with $p_{i} p_{j} \geq 0$ and summing over $i$ and $j$ from 1 to $n$, we get

$$
\begin{aligned}
& \sum_{i=1}^{n} p_{i} \nabla_{-} f\left(u_{i}\right)\left(v_{i}\right)+\sum_{j=1}^{n} p_{j} \nabla_{+} f\left(u_{j}\right)\left(v_{j}\right) \\
& \quad \geq \sum_{i, j=1}^{n} p_{i} p_{j} \nabla_{+} f\left(u_{j}\right)\left(v_{i}\right)+\sum_{i, j=1}^{n} p_{i} p_{j} \nabla_{-} f\left(u_{i}\right)\left(v_{j}\right)
\end{aligned}
$$

Now, observe that

$$
\sum_{j=1}^{n} p_{j} \nabla_{+} f\left(u_{j}\right)\left(v_{j}\right)=\sum_{i=1}^{n} p_{i} \nabla_{+} f\left(u_{i}\right)\left(v_{i}\right)
$$

and

$$
\sum_{i, j=1}^{n} p_{i} p_{j} \nabla_{+} f\left(u_{j}\right)\left(v_{i}\right)=\sum_{i, j=1}^{n} p_{i} p_{j} \nabla_{+} f\left(u_{i}\right)\left(v_{j}\right),
$$

which, by (2.14) divided by 2 , provides the desired result (2.10).

COROLlary 2.3. With the assumptions of Theorem 2.2, and if in addition $\tilde{\nabla} f\left(u_{i}\right)(\cdot)$ is additive for any $i \in\{1, \ldots, n\}$, then

$$
\sum_{i=1}^{n} p_{i} \tilde{\nabla} f\left(u_{i}\right)\left(v_{i}\right) \geq \sum_{i=1}^{n} p_{i} \tilde{\nabla} f\left(u_{i}\right)\left(\sum_{j=1}^{n} p_{j} u_{j}\right) .
$$

REMARK 2.4. If $f$ is Gâteaux differentiable at the points $u_{i}, i \in\{1, \ldots, n\}$, then $\tilde{\nabla} f\left(u_{i}\right)(\cdot)=\nabla f\left(u_{i}\right)(\cdot)$ and is therefore linear on $X$. With this assumption, inequality (2.15) becomes

$$
\sum_{i=1}^{n} p_{i} \nabla f\left(u_{i}\right)\left(v_{i}\right) \geq \sum_{i=1}^{n} p_{i} \nabla f\left(u_{i}\right)\left(\sum_{j=1}^{n} p_{j} u_{j}\right) .
$$


Moreover, we note that there are examples of convex functions defined on linear spaces for which $\tilde{\nabla} f(x)(\cdot)$ is linear for any $x \neq 0$ without the function $f$ being Gâteaux differentiable at that point (see, for instance, [10, pp. 44-45]).

Following [19], we consider the $g$-semi-inner product $\langle\cdot, \cdot\rangle_{g}: X \times X \rightarrow \mathbb{R}$ defined by

$$
\langle y, x\rangle_{g}:=\frac{1}{2}\left[\langle y, x\rangle_{i}+\langle y, x\rangle_{s}\right], \quad x, y \in X .
$$

Using this notation, we have the following conditional inequality for semi-inner products and norms in normed linear spaces.

Proposition 2.5. Let $(X,\|\cdot\|)$ be a normed linear space, $u=\left(u_{1}, \ldots, u_{n}\right), v=$ $\left(v_{1}, \ldots, v_{n}\right) \in X^{n}$ and $p \geq 1$. If

$$
\left\|u_{k}\right\|^{p-2}\left\langle v_{k}-v_{j}, u_{k}\right\rangle_{i} \geq\left\|u_{j}\right\|^{p-2}\left\langle v_{k}-v_{j}, u_{j}\right\rangle_{s}
$$

for any $k, j \in\{1, \ldots, n\}$, then

$$
\sum_{k=1}^{n} p_{k}\left\|u_{k}\right\|^{p-2}\left\langle v_{k}, u_{k}\right\rangle_{g} \geq \sum_{k, j=1}^{n} p_{k} p_{j}\left\|u_{k}\right\|^{p-2}\left\langle v_{j}, u_{k}\right\rangle_{g}
$$

for any $\mathbf{p}$ a probability distribution. If $p<2$, then we should have in (2.17) all $u_{k} \neq 0$. If $p=2$ and

$$
\left\langle v_{k}-v_{j}, u_{k}\right\rangle_{i} \geq\left\langle v_{k}-v_{j}, u_{j}\right\rangle_{s}
$$

for any $k, j \in\{1, \ldots, n\}$, then

$$
\sum_{k=1}^{n} p_{k}\left\langle v_{k}, u_{k}\right\rangle_{g} \geq \sum_{k, j=1}^{n} p_{k} p_{j}\left\langle v_{j}, u_{k}\right\rangle_{g}
$$

for any $\mathbf{p}$ a probability distribution.

As a particular case of interest, we state the following result that holds in inner product spaces.

COROLlaRY 2.6. Let $(X,\langle\cdot, \cdot\rangle)$ be a real inner product space, $u=\left(u_{1}, \ldots, u_{n}\right)$, $v=\left(v_{1}, \ldots, v_{n}\right) \in X^{n}$ and $p \geq 1$. If

$$
\left\langle v_{k}-v_{j},\left\|u_{k}\right\|^{p-2} u_{k}-\left\|u_{j}\right\|^{p-2} u_{j}\right\rangle \geq 0
$$

for any $k, j \in\{1, \ldots, n\}$, then

$$
\sum_{k=1}^{n} p_{k}\left\|u_{k}\right\|^{p-2}\left\langle v_{k}, u_{k}\right\rangle \geq\left\langle\sum_{j=1}^{n} p_{j} u_{j}, \sum_{k=1}^{n} p_{k}\left\|u_{k}\right\|^{p-2} u_{k}\right\rangle
$$

for any $\mathbf{p}$ a probability distribution.

REMARK 2.7. We observe that if the $n$-tuples $u$ and $v$ above are synchronous, that is,

$$
\left\langle v_{k}-v_{j}, u_{k}-u_{j}\right\rangle \geq 0 \text { for any } j, k \in\{1, \ldots, n\},
$$


then we have the Chebyshev type inequality

$$
\sum_{k=1}^{n} p_{k}\left\langle v_{k}, u_{k}\right\rangle \geq\left\langle\sum_{k=1}^{n} p_{k} v_{k}, \sum_{k=1}^{n} p_{k} u_{k}\right\rangle .
$$

This result was first obtained in [18].

\section{Inequalities for convex functions}

The following result for convex functions may be stated.

THEOREM 3.1. Let $f: X \rightarrow \mathbb{R}$ be a convex function on the linear space $X$ and $x, y \in X^{n}$. Let $\mathbf{p}$ be a probability distribution so that

$$
\sum_{i=1}^{n} p_{i} x_{i}=\sum_{i=1}^{n} p_{i} y_{i}
$$

If $x-y$ is $\nabla-f$-synchronous with $y$ and $\tilde{\nabla} f\left(y_{i}\right)(\cdot)$ is additive for each $i \in$ $\{1, \ldots, n\}$, then

$$
\sum_{i=1}^{n} p_{i} f\left(x_{i}\right) \geq \sum_{i=1}^{n} p_{i} f\left(y_{i}\right)
$$

Proof. Since $f$ is convex, then for any $x, y \in X$,

$$
f(x)-f(y) \geq \nabla_{+} f(y)(x-y) \geq \tilde{\nabla} f(y)(x-y) .
$$

Then from (3.2),

$$
f\left(x_{i}\right)-f\left(y_{i}\right) \geq \tilde{\nabla} f\left(y_{i}\right)\left(x_{i}-y_{i}\right)
$$

for each $i \in\{1, \ldots, n\}$.

If we multiply (3.3) by $p_{i} \geq 0$ and then sum over $i$ from 1 to $n$, we get

$$
\sum_{i=1}^{n} p_{i} f\left(x_{i}\right)-\sum_{i=1}^{n} p_{i} f\left(y_{i}\right) \geq \sum_{i=1}^{n} p_{i} \tilde{\nabla} f\left(y_{i}\right)\left(x_{i}-y_{i}\right) .
$$

If we now use Corollary 2.3 for $u_{i}=y_{i}$ and $v_{i}=x_{i}-y_{i}, i \in\{1, \ldots, n\}$, we deduce the inequality

$$
\begin{aligned}
\sum_{i=1}^{n} p_{i} \tilde{\nabla} f\left(y_{i}\right)\left(x_{i}-y_{i}\right) & \geq \sum_{i=1}^{n} p_{i} \tilde{\nabla} f\left(y_{i}\right)\left(\sum_{i=1}^{n} p_{i}\left(x_{i}-y_{i}\right)\right) \\
& =\sum_{i=1}^{n} p_{i} \tilde{\nabla} f\left(y_{i}\right)(0)=0 .
\end{aligned}
$$

Combining (3.4) with (3.5), we deduce the desired inequality (3.1).

REMARK 3.2. It is clear that if $f$ is Gâteaux differentiable at all the points $y_{i}$, $i \in\{1, \ldots, n\}$, then $\tilde{\nabla} f\left(y_{i}\right)(\cdot)=\nabla f\left(y_{i}\right)(\cdot), i \in\{1, \ldots, n\}$, which are linear on $X$ and then inequality (3.1) holds true. 
In the case of Gâteaux differentiable functions, we can state the following result as well.

THEOREM 3.3. Let $f: X \rightarrow \mathbb{R}$ be a convex and Gâteaux differentiable function on the linear space $X$. Assume that $x, y \in X^{n}$ and $\mathbf{p}$ is a probability distribution. If $x-y$ is $\nabla-f$-synchronous with $y$ and

$$
\sum_{i=1}^{n} p_{i} x_{i}-\sum_{i=1}^{n} p_{i} y_{i} \in \bigcap_{i=1}^{n} \operatorname{ker}\left(\nabla f\left(y_{i}\right)(\cdot)\right),
$$

then

$$
\sum_{i=1}^{n} p_{i} f\left(x_{i}\right) \geq \sum_{i=1}^{n} p_{i} f\left(y_{i}\right) .
$$

The proof is as for Theorem 3.1 when in (3.5) we take into account that

$$
\nabla f\left(y_{i}\right)\left(\sum_{i=1}^{n} p_{i} x_{i}-\sum_{i=1}^{n} p_{i} y_{i}\right)=0
$$

for all $i \in\{1, \ldots, n\}$ since

$$
\sum_{i=1}^{n} p_{i} x_{i}-\sum_{i=1}^{n} p_{i} y_{i} \in \bigcap_{i=1}^{n} \operatorname{ker}\left(\nabla f\left(y_{i}\right)(\cdot)\right) .
$$

The following result in smooth normed linear spaces may be stated.

Proposition 3.4. Let $(X,\|\cdot\|)$ be a smooth normed linear space and let $[\cdot, \cdot]$ be the semi-inner product that generates its norm $\|\cdot\|$. If $x, y \in X^{n}$ and $p \geq 1$ are such that

$$
\left\|y_{k}\right\|^{p-2}\left[x_{k}-y_{k}-x_{j}+y_{j}, y_{k}\right] \geq\left\|y_{j}\right\|^{p-2}\left[x_{k}-y_{k}-x_{j}+y_{j}, y_{j}\right]
$$

for any $k, j \in\{1, \ldots, n\}$, then, for any probability distribution $\mathbf{p}$ with the property that

$$
\sum_{j=1}^{n} p_{j} x_{j}=\sum_{j=1}^{n} p_{j} y_{j}
$$

we have the inequality

$$
\sum_{k=1}^{n} p_{k}\left\|x_{k}\right\|^{p} \geq \sum_{k=1}^{n} p_{k}\left\|y_{k}\right\|^{p}
$$

If $p \in[1,2)$ we shall assume that $y_{k} \neq 0$ for $k \in\{1, \ldots, n\}$.

If $p=2$ and

$$
\left[x_{k}-y_{k}-x_{j}+y_{j}, y_{k}\right] \geq\left[x_{k}-y_{k}-x_{j}+y_{j}, y_{j}\right]
$$

for any $k, j \in\{1, \ldots, n\}$, then for any probability distribution $\mathbf{p}$ satisfying (3.8),

$$
\sum_{k=1}^{n} p_{k}\left\|x_{k}\right\|^{2} \geq \sum_{k=1}^{n} p_{k}\left\|y_{k}\right\|^{2}
$$


The case of inner product spaces is incorporated in the following corollary.

Corollary 3.5. Let $(X ;\langle\cdot, \cdot\rangle)$ be an inner product space. If $x, y \in X^{n}$ and $p \geq 1$ are such that

$$
\left\langle x_{k}-x_{j},\left\|y_{k}\right\|^{p-2} y_{k}-\left\|y_{j}\right\|^{p-2} y_{j}\right\rangle \geq\left\langle y_{k}-y_{j},\left\|y_{k}\right\|^{p-2} y_{k}-\left\|y_{j}\right\|^{p-2} y_{j}\right\rangle
$$

for any $k, j \in\{1, \ldots, n\}$, then for any $\mathbf{p}$ satisfying (3.8) we have inequality (3.9).

If $p \in[1,2)$, then we shall assume that $y_{k} \neq 0, k \in\{1, \ldots, n\}$.

If $p=2$ and

$$
\left\langle x_{k}-x_{j}, y_{k}-y_{j}\right\rangle \geq\left\|y_{k}-y_{j}\right\|^{2}, \quad \text { for any } k, j \in\{1, \ldots, n\},
$$

then for any $\mathbf{p}$ satisfying (3.8), we have inequality (3.11).

REMARK 3.6. We notice that examples of sequences $x_{k}$ and $y_{k}, k \in\{1, \ldots, n\}$, satisfying (3.13) can easily be provided by taking $x_{k}=A y_{k}, k \in\{1, \ldots, n\}$, where $A$ is a selfadjoint operator on the Hilbert space $H$ satisfying the condition $\langle A z, z\rangle \geq\|z\|^{2}$, for any $z \in H$, that is, $A \geq I$ in the operator order of the Banach algebra $B(H)$.

\section{Applications for convex functions on $\mathbb{R}^{m}$}

Now consider an open and convex set $C$ in the real linear space $\mathbb{R}^{m}, m \geq 1$. For a convex and differentiable function $f: C \rightarrow \mathbb{R}$,

$$
\nabla f(x)(y)=\left\langle\frac{\partial f(x)}{\partial x}, y\right\rangle, \quad x \in C, y \in \mathbb{R}^{m},
$$

where

$$
\frac{\partial f(x)}{\partial x}=\left(\frac{\partial f(x)}{\partial x^{1}}, \ldots, \frac{\partial f(x)}{\partial x^{m}}\right), \quad x=\left(x^{1}, \ldots, x^{m}\right) \in C
$$

and $\langle\cdot, \cdot\rangle$ is the usual inner product in $\mathbb{R}^{m}$, that is, $\langle u, v\rangle=\sum_{k=1}^{m} u^{i} \cdot v^{i}$, where $u=\left(u^{1}, \ldots, u^{m}\right)$ and $v=\left(v^{1}, \ldots, v^{m}\right) \in \mathbb{R}^{m}$.

Now, if $\mathbf{v}:=\left(v_{1}, \ldots, v_{n}\right) \in \mathbb{R}^{m}$ and $\mathbf{u}:=\left(u_{1}, \ldots, u_{n}\right) \in C^{m}$, then we say that $\mathbf{v}$ is $\nabla-f$-synchronous with $\mathbf{u}$ if

$$
\left\langle\frac{\partial f\left(u_{k}\right)}{\partial x}-\frac{\partial f\left(u_{j}\right)}{\partial x}, v_{k}-v_{j}\right\rangle \geq 0 \text { for any } k, j \in\{1, \ldots, n\} .
$$

The following result may be stated.

Proposition 4.1. Let $f: C \rightarrow \mathbb{R}$ be a differentiable convex function on the open and convex set $C \subseteq \mathbb{R}^{m}$. If $\mathbf{v}:=\left(v_{1}, \ldots, v_{n}\right) \in \mathbb{R}^{m}$ and $\mathbf{u}:=\left(u_{1}, \ldots, u_{n}\right) \in C^{m}$ are such that $\mathbf{v}$ is $\nabla-f$-synchronous with $\mathbf{u}$, then for any probability distribution $\mathbf{p}=\left(p_{1}, \ldots, p_{n}\right)$,

$$
\sum_{i=1}^{n} p_{i}\left\langle\frac{\partial f\left(u_{i}\right)}{\partial x}, v_{i}\right\rangle \geq\left\langle\sum_{i=1}^{n} p_{i} \frac{\partial f\left(u_{i}\right)}{\partial x}, \sum_{i=1}^{n} p_{i} v_{i}\right\rangle .
$$

The proof is obvious by Corollary 2.3. 
Now, if $u_{k}=\left(u_{k}^{1}, \ldots, u_{k}^{m}\right), k \in\{1, \ldots, n\}$, and $v_{k}=\left(v_{k}^{1}, \ldots, v_{k}^{m}\right)$, then

$$
\left\langle\frac{\partial f\left(u_{k}\right)}{\partial x}-\frac{\partial f\left(u_{j}\right)}{\partial x}, v_{k}-v_{j}\right\rangle=\sum_{\ell=1}^{m}\left(\frac{\partial f\left(u_{k}\right)}{\partial x^{\ell}}-\frac{\partial f\left(u_{j}\right)}{\partial x^{\ell}}\right)\left(v_{k}^{\ell}-v_{j}^{\ell}\right) .
$$

REMARK 4.2. Relation (4.4) shows that a sufficient condition for $\mathbf{v}$ to be $\nabla-f$ synchronous with $\mathbf{u}$ is that all the sequences $\left\{\partial f\left(u_{k}\right) / \partial x^{\ell}\right\}_{k=1, \ldots, n}$ and $\left\{v_{k}^{\ell}\right\}_{k=1, \ldots, n}$ are synchronous, where $\ell \in\{1, \ldots, m\}$, that is,

$$
\left(\frac{\partial f\left(u_{k}\right)}{\partial x^{\ell}}-\frac{\partial f\left(u_{j}\right)}{\partial x^{\ell}}\right)\left(v_{k}^{\ell}-v_{j}^{\ell}\right) \geq 0 \text { for any } k, j \in\{1, \ldots, n\}
$$

and for all $\ell \in\{1, \ldots, m\}$.

The following result is an obvious consequence of Theorem 3.3.

Proposition 4.3. Let $f: C \rightarrow \mathbb{R}$ be a differentiable convex function on the open and convex set $C \subseteq \mathbb{R}^{m}$. If $\mathbf{x}=\left(x_{1}, \ldots, x_{n}\right) \in \mathbb{R}^{m}$ and $\mathbf{y}=\left(y_{1}, \ldots, y_{n}\right) \in C^{m}$ are such that

$$
\left\langle\frac{\partial f\left(y_{k}\right)}{\partial x}-\frac{\partial f\left(y_{j}\right)}{\partial x}, x_{k}-x_{j}\right\rangle \geq\left\langle\frac{\partial f\left(y_{k}\right)}{\partial x}-\frac{\partial f\left(y_{j}\right)}{\partial x}, y_{k}-y_{j}\right\rangle,
$$

for each $k, j \in\{1, \ldots, n\}$, then for any probability distribution $\mathbf{p}=\left(p_{1}, \ldots, p_{n}\right)$ with

$$
\sum_{i=1}^{n} p_{i} x_{i}=\sum_{i=1}^{n} p_{i} y_{i}
$$

we have the inequality

$$
\sum_{i=1}^{n} p_{i} f\left(x_{i}\right) \geq \sum_{i=1}^{n} p_{i} f\left(y_{i}\right) .
$$

REMARK 4.4. As above, a sufficient condition for (4.6) to hold is that the sequences $\left\{\partial f\left(y_{k}\right) / \partial x^{\ell}\right\}_{k=1, \ldots, n}$ and $\left\{x_{k}^{\ell}-y_{k}^{\ell}\right\}_{k=1, \ldots, n}$ are synchronous for each $\ell \in\{1, \ldots, m\}$.

\section{References}

[1] A. H. Ansari and M. S. Moslehian, 'More on reverse triangle inequality in inner product spaces', Int. J. Math. Math. Sci. 2005(18) (2005), 2883-2893.

[2] A. H. Ansari and M. S. Moslehian, 'Refinements of reverse triangle inequalities in inner product spaces', J. Inequal. Pure Appl. Math. 6(3) (2005), Article 64, 12 pp (electronic).

[3] I. Brnetić, S. S. Dragomir, R. Hoxha and J. Pečarić, 'A reverse of the triangle inequality in inner product spaces and applications for polynomials', Aust. J. Math. Anal. Appl. 3(2) (2006), Art. 9, $8 \mathrm{pp}$.

[4] Y. J. Cho, M. Matić and J. Pečarić, 'Inequalities of Hlawka's type in $n$-inner product spaces', Commun. Korean Math. Soc. 17(4) (2002), 583-592.

[5] S. S. Dragomir, 'An improvement of Jensen's inequality', Bull. Math. Soc. Sci. Math. Roumanie 34(82)(4) (1990), 291-296.

[6] S. S. Dragomir, 'Some refinements of Ky Fan's inequality', J. Math. Anal. Appl. 163(2) (1992), 317-321.

[7] S. S. Dragomir, 'Some refinements of Jensen's inequality', J. Math. Anal. Appl. 168(2) (1992), 518-522. 
[8] S. S. Dragomir, 'A further improvement of Jensen's inequality', Tamkang J. Math. 25(1) (1994), 29-36.

[9] S. S. Dragomir, 'A new improvement of Jensen's inequality', Indian J. Pure Appl. Math. 26(10) (1995), 959-968.

[10] S. S. Dragomir, Semi-inner Products and Applications (Nova Science Publishers, New York, 2004).

[11] S. S. Dragomir, 'A new refinement of Jensen's inequality in linear spaces with applications', RGMIA Res. Rep. Coll. 12 (2009), Preprint, Supplement, Article 6. http://www.staff.vu.edu.au/RGMIA/v12(E).asp.

[12] S. S. Dragomir, 'Inequalities in terms of the Gâteaux derivatives for convex functions in linear spaces with applications', RGMIA Res. Rep. Coll. 12 (2009), Preprint, Supplement, Article 7. http://www.staff.vu.edu.au/RGMIA/v12(E).asp.

[13] S. S. Dragomir, 'A refinement of Jensen's inequality with applications for $f$-divergence measures', Taiwanese J. Math. 14(1) (2010), 153-164.

[14] S. S. Dragomir and C. J. Goh, 'A counterpart of Jensen's discrete inequality for differentiable convex mappings and applications in information theory', Math. Comput. Modelling 24(2) (1996), $1-11$.

[15] S. S. Dragomir and N. M. Ionescu, 'Some converse of Jensen's inequality and applications', Rev. Anal. Numér. Théor. Approx. 23(1) (1994), 71-78.

[16] S. S. Dragomir, J. Pečarić and L. E. Persson, 'Properties of some functionals related to Jensen's inequality', Acta Math. Hungar. 70(1-2) (1996), 129-143.

[17] S. S. Dragomir, J. Pečarić and J. Sándor, 'The Chebyshev inequality in pre-Hilbertian spaces. II', Proceedings of the Third Symposium of Mathematics and its Applications (Timişoara, 1989) (Romanian Acad., Timişoara, 1990), pp. 75-78.

[18] S. S. Dragomir and J. Sándor, 'The Chebyshev inequality in pre-Hilbertian spaces. I', Proceedings of the Second Symposium of Mathematics and its Applications (Timişoara, 1987) (Romanian Acad., Timişoara, 1988), pp. 61-64.

[19] P. M. Miličić, 'Sur le semi-produit scalaire dans quelques espaces vectorial normés', Mat. Vesnik 8(23) (1971), 181-185.

[20] P. M. Miličić, 'Sur une inégalité complémentaire de l'inégalité triangulaire', Mat. Vesnik 41(2) (1989), 83-88.

[21] J. Pečarić and S. S. Dragomir, 'A refinements of Jensen inequality and applications', Stud. Univ. Babeş-Bolyai, Math. 24(1) (1989), 15-19.

[22] J. Pečarić and R. Rajić, 'The Dunkl-Williams inequality with $n$ elements in normed linear spaces', Math. Inequal. Appl. 10(2) (2007), 461-470.

S. S. DRAGOMIR, Mathematics, School of Engineering and Science,

Victoria University, PO Box 14428, Melbourne City,

Vic 8001, Australia

and

School of Computational and Applied Mathematics, University of the Witwatersrand, Private Bag-3, Wits-2050, Johannesburg, South Africa

e-mail: sever.dragomir@vu.edu.au 